07

\title{
Измерение внутреннего квантового выхода излучения InGaN светодиода
}

\author{
() И.В. Фролов, ${ }^{1}$ В.А. Сергеев, ${ }^{1,2}$ О.А. Радаев ${ }^{1}$ \\ ${ }^{1}$ Ульяновский фрилиал Института радиотехники и электроники им. В.А. Котельникова РАН, \\ 432071 Ульяновск, Россия \\ ${ }^{2}$ Ульяновский государственный технический университет, \\ 432026 Ульяновск, Россия \\ e-mail: ilya-frolov88@mail.ru
}

Поступило в Редакцию 4 марта 2020 г.

В окончательной редакции 25 марта 2020 г.

Принято к публикации 26 марта 2020 г.

\begin{abstract}
Представлен способ измерения внутреннего квантового выхода излучения InGaN светодиода, заключающийся в измерении мощности излучения и граничных частот электролюминесценции светодиода при двух малых токах, соответствующих участку роста квантовой эффективности светодиода, и расчете значения внутреннего квантового выхода по соответствующей функциональной зависимости. Для определения внутреннего квантового выхода при других значениях тока измерена токовая зависимость внешнего квантового выхода и рассчитан коэффициент вывода излучения из структуры по результатам измерений внутреннего и внешнего квантового выхода при малом токе. Достоверность способа измерений подтверждена сопоставлением результатов измерений с результатами, полученными известным способом измерений.
\end{abstract}

Ключевые слова: светодиод, внутренний квантовый выход, измерение.

DOI: $10.21883 /$ JTF.2021.08.51102.54-21

\section{Введение}

Важнейшим параметром светодиодов, определяющим эффективность преобразования электрического тока в оптическое излучение, является внутренний квантовый выход. Этот параметр используется при оценке качества изготовления светодиодных гетероструктур, их энергетической эффективности, а также при исследовании механизмов спада эффективности излучения при больших токах и механизмов деградации светодиодов в процессе их испытаний [1-5]. Внутренний квантовый выход определяется как отношение числа фотонов, рожденных в активной области светодиода в единицу времени, к числу инжектированных в эту область электронов [1].

Большинство известных методов измерения внутреннего квантового выхода основаны на измерении параметров фотолюминесценции [5-7] или электролюминесценции [8] светодиода при температурах 4-10 K. При peaлизации этих способов предполагается, что при криогенных температурах центры безызлучательной рекомбинации в активной области гетероструктуры светодиода становятся неактивными, а скорость оже-рекомбинации, которая экспоненциально уменьшается с понижением температуры, становится пренебрежимо малой. Поэтому квантовая эффективность излучения при криогенных температурах считается равной $100 \%$ [1]. Для определения значения внутреннего квантового выхода светодиода при других температурах зависимости квантовой эффективности нормируются на значение эффективности, полученное при криогенной температуре.
Также известен метод, основанный на измерении внутреннего квантового выхода по параметрам катодолюминесценции светодиодной гетероструктуры [9]. Метод подразумевает облучение структуры пучком электронов в импульсном режиме и измерение интенсивности катодолюминесценции. При этом абсолютное значение внутреннего квантового выхода тестируемых гетероструктур определяется путем нормирования интенсивности излучения на интенсивность излучения эталонного образца с известным абсолютным значением внутреннего квантового выхода.

Указанные методы измерений характеризуются сложностью аппаратной реализации или необходимостью наличия эталонного образца, что затрудняет их применение для определения внутреннего квантового выхода излучения светодиода в рабочих условиях эксплуатации. В настоящей работе представлен способ измерения, позволяющий определять внутренний квантовый выход излучения InGaN светодиода и коэффициент вывода излучения из структуры при любых температурах, в том числе комнатной, не требующий наличия образцового светодиода с известными параметрами.

\section{1. Описание способа измерения внутреннего квантового выхода излучения}

В основе способа измерения [10] лежит общепринятая $\mathrm{ABC}$-модель рекомбинации носителей заряда в гетероструктуре $\mathrm{InGaN}$ светодиода, устанавливающая связь 
внутреннего квантового выхода излучения светодиода с концентрацией носителей заряда в активной области через рекомбинационные коэффициенты. При отсутствии утечки носителей заряда из активной области гетероструктуры выражение для внутреннего квантового выхода светодиода $\eta$ можно записать в виде [11]

$$
\eta=\frac{B n^{2}}{A n+B n^{2}+C n^{3}},
$$

где $A-$ коэффициент безызлучательной рекомбинации по механизму Шокли-Рида-Холла, $B$ - коэффициент излучательной рекомбинации, $C-$ коэффициент оже-рекомбинации, $n$ - концентрация носителей заряда в активной области гетероструктуры, определяемая плотностью рабочего тока.

В свою очередь, с рекомбинационными коэффициентами $A, B, C$ и концентрацией n носителей заряда в активной области связано дифференциальное время жизни носителей заряда $\tau$ [12]:

$$
\tau^{-1}=A+2 B n+3 C n^{2} .
$$

При малых токах, соответствующих диапазону роста внешней квантовой эффективности светодиода, влиянием оже-рекомбинации можно пренебречь $\left(\mathrm{Cn}^{3} \approx 0\right)$. В этом случае

$$
\begin{gathered}
\eta \approx \frac{B n^{2}}{A n+B n^{2}}, \\
\tau^{-1} \approx A+2 B n .
\end{gathered}
$$

Объединяя (3) и (4) и исключая коэффициент излучательной рекомбинации $B$, получим

$$
\eta=\frac{1-A \tau}{1+A \tau}
$$

Полная мощность оптического излучения $P$, выходящего их светодиода, зависит от коэффициента оптического вывода излучения $\eta_{\text {extr }}$ и определяется выражением [13]

$$
P=\eta_{\text {extr }} V \frac{h c}{\lambda} B n^{2},
$$

где $V$ - объем активной области, $\lambda-$ длина волны излучения, $h-$ постоянная Планка, $c-$ скорость света в вакууме.

Поскольку коэффициент $\eta_{\text {extr }}$ в основном определяется свойствами материалов, из которых изготовлен светодиод, и конструкцией светодиода и слабо зависит от тока, протекающего через светодиод, для двух значений концентраций носителей заряда $n_{1}$ и $n_{2}$, соответствующих заданным значениям электрического тока $I_{1}$ и $I_{2}$, можно составить систему уравнений:

$$
\left\{\begin{array}{l}
P\left(I_{1}\right)=\eta_{\text {extr }} V \frac{h c}{\lambda} B n\left(I_{1}\right)^{2} \\
P\left(I_{2}\right)=\eta_{\text {extr }} V \frac{h c}{\lambda} B n\left(I_{2}\right)^{2} \\
\tau\left(I_{1}\right)^{-1}=A+2 B n\left(I_{1}\right) \\
\tau\left(I_{2}\right)^{-1}=A+2 B n\left(I_{2}\right)
\end{array}\right.
$$

где $P\left(I_{1}\right)$ и $P\left(I_{2}\right)$ - полная мощность излучения светодиода, измеренная при токах $I_{1}$ и $I_{2}$ соответственно (причем $I_{2}>I_{1}$ ).

Решая систему уравнений (7) относительно коэффициента $A$, получим

$$
A=\frac{1}{\tau\left(I_{1}\right)}\left(1-\frac{\tau\left(I_{1}\right) / \tau\left(I_{2}\right)-1}{\sqrt{P\left(I_{2}\right) / P\left(I_{1}\right)}-1}\right) .
$$

Подставляя (8) в (5), получим общее выражение для нахождения внутреннего квантового выхода $\eta$ светодиода при произвольном значении тока $I$ :

$$
\eta(I)=\frac{1-\frac{\tau(I)}{\tau\left(I_{1}\right)}\left(1-\frac{\tau\left(I_{1}\right) / \tau\left(I_{2}\right)-1}{\sqrt{P\left(I_{2}\right) / P\left(I_{1}\right)}-1}\right)}{1+\frac{\tau(I)}{\tau\left(I_{1}\right)}\left(1-\frac{\tau\left(I_{1}\right) / \tau\left(I_{2}\right)-1}{\sqrt{P\left(I_{2}\right) / P\left(I_{1}\right)}-1}\right)} .
$$

Поскольку дифференциальное время жизни носителей заряда $\tau$ определяет граничную частоту $f_{3 d B}$ модуляции электролюминесценции светодиода $[12,13]$

$$
f_{3 d B}=\frac{\sqrt{3}}{2 \pi \tau}
$$

выражение (9) для внутреннего квантового выхода при произвольном токе $I$ примет вид

$$
\eta(I)=\frac{1-\frac{f_{3 d B}\left(I_{1}\right)}{f_{3 d B}(I)}\left(1-\frac{f_{3 d B}\left(I_{2}\right) / f_{3 d B}\left(I_{1}\right)-1}{\sqrt{P\left(I_{2}\right) / P\left(I_{1}\right)}-1}\right)}{1+\frac{f_{3 d B}\left(I_{1}\right)}{f_{3 d B}(I)}\left(1-\frac{f_{3 d B}\left(I_{2}\right) / f_{3 d B}\left(I_{1}\right)-1}{\sqrt{P\left(I_{2}\right) / P\left(I_{1}\right)}-1}\right)} .
$$

Погрешность измерения внутреннего квантового выхода определяется погрешностями измерений граничных частот электролюминесценции и мощности излучения. Так как максимум квантовой эффективности InGaN светодиодов достигается при плотностях тока порядка $1-10 \mathrm{~A} / \mathrm{cm}^{2}$, измерения величин, входящих в выражение (11), для маломощных светодиодов связаны c регистрацией слабых переменных оптических сигналов. Поскольку современные ваттметры позволяют измерять мощность постоянного излучения с довольно высокой точностью, погрешность измерения $\eta(I)$ преимущественно определяется погрешностью измерения граничной частоты $f_{3 d B}$, которая зависит от уровня шума и чувствительности фотоприемника. Поэтому выбор значения тока $I_{1}$ для измерения мощности излучения и граничной частоты модуляции определяется точностью средств измерений, а $I_{1}$ выбирается наименьшим, при котором обеспечивается заданный уровень точности. Ток $I_{2}$ выбирается из диапазона значений, при которых токовая зависимость квантового выхода возрастает и вкладом оже-рекомбинационной составляющей в формирование значения внутреннего квантового выхода можно пренебречь. Для выполнения этого условия ток $I_{2}$ должен быть в 5-10 раз меньше, чем ток, при котором достигается максимум зависимости $\eta(I)$. 
Поскольку внешний $\eta_{\text {ext }}$ и внутренний $\eta$ квантовые выходы связаны между собой через коэффициент вывода излучения $\eta_{\text {extr }}$, для определения $\eta_{\text {extr }}$ достаточно измерить значения $\eta_{\operatorname{ext}\left(I_{1}\right)}$ и $\eta\left(I_{1}\right)$ при токе $I_{1}$ и вычислить их отношение:

$$
\eta_{\text {extr }}=\frac{\eta_{\text {ext }}\left(I_{1}\right)}{\eta\left(I_{1}\right)}
$$

В свою очередь, внешний квантовый выход определяется по результатам измерения мощности излучения светодиода при токе $I_{1}$ путем расчета по формуле [13]

$$
\eta_{e x t}=\frac{P\left(I_{1}\right)}{I_{1}} \frac{q \lambda}{h c}
$$

где $q-$ заряд электрона.

При известных значениях коэффициента вывода излучения и токовой зависимости внешней квантовой эффективности можно определить внутренний квантовый выход при любом токе светодиода.

\section{2. Экспериментальная апробация способа измерения}

Способ измерения апробирован на примере коммерческого зеленого светодиода C503B-GAN фирмы Cree, имеющего следующие параметры: длина волны максимума спектра излучения $527 \mathrm{~nm}$ при токе $20 \mathrm{~mA}$; номинальный рабочий ток $20 \mathrm{~mA}$; максимальный ток $30 \mathrm{~mA}$; размеры кристалла $250 \times 420 \mu \mathrm{m}$.

Ватт-амперная характеристика светодиода измерялась интегрирующей сферой TES-133 в диапазоне токов $10 \mu \mathrm{A}-20 \mathrm{~mA}$. Внешний квантовый выход излучения рассчитывался по формуле (13). Максимальное значение функция $\eta_{\text {ext }}(I)$ достигает при токе $700 \mu \mathrm{A}$. График представлен на рис. 1 .

Для определения внутреннего квантового выхода были измерены граничные частоты электролюминесценции при токах $I_{1}=10 \mu \mathrm{A}$ и $I_{2}=50 \mu \mathrm{A}$. При измерениях через светодиод пропускались постоянный ток и гармонический ток малой амплитуды. Для минимизации влияния нелинейности вольт-амперной и ватт-амперной

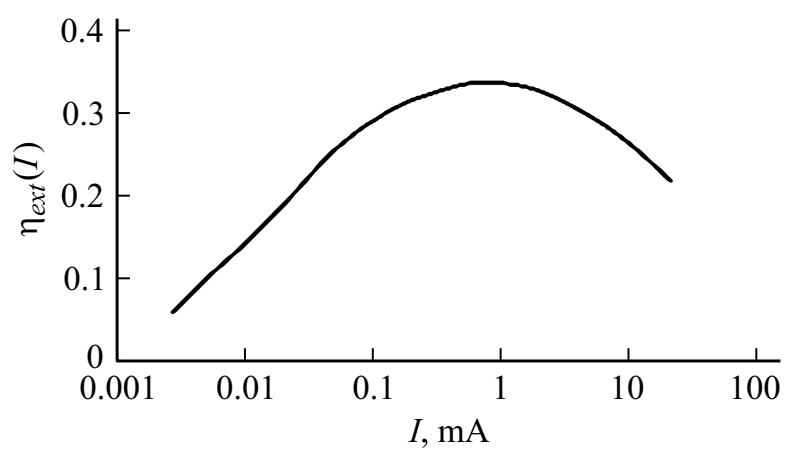

Рис. 1. Зависимость внешнего квантового выхода излучения светодиода от тока.
Таблица 1. Результаты измерений внутреннего квантового выхода

\begin{tabular}{c|c|c|c|c|c}
\hline$I, \mu \mathrm{A}$ & $f_{3 d B}(I), \mathrm{kHz}$ & $P, \mu \mathrm{W}$ & $\eta(I)$ & $\eta_{\text {ext }}(I)$ & $\eta_{\text {extr }}$ \\
\hline 10 & 290 & 3.45 & 0.312 & 0.148 & \multirow{2}{*}{0.47} \\
\cline { 1 - 5 } 50 & 560 & 30.2 & 0.573 & 0.270 &
\end{tabular}

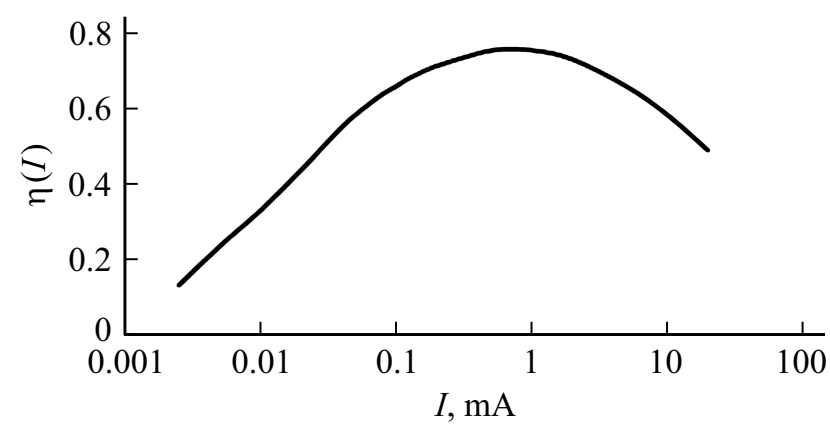

Рис. 2. Зависимость внутреннего квантового выхода излучения светодиода от тока.

характеристик светодиода на результат измерений амплитуда переменной составляющей тока задавалась равной $10 \%$ от значения постоянной составляющей сигнала в соответствии с требованиями ГОСТ 18986.14-85. В начале измерений частота сигнала устанавливалась равной $1 \mathrm{kHz}$. Переменная составляющая сигнала оптического излучения светодиода регистрировалась широкополосным фотоприемником с равномерной амплитудночастотной характеристикой в полосе частот до $10 \mathrm{MHz}$. Амплитуда гармоники определялась с помощью преобразования Фурье. Затем частота тока светодиода увеличивалась с равномерным шагом по логарифмической шкале. Граничная частота электролюминесценции определялась как частота, при которой амплитуда основной гармоники переменного оптического излучения уменьшалась в два раза относительно значения, измеренного на частоте $1 \mathrm{kHz}$. После расчета внутреннего квантового выхода при токах $I_{1}$ и $I_{2}$ в соответствии с выражением (11) рассчитывался коэффициент вывода излучения $\eta_{\text {extr }}$ по формуле (12). Результаты измерений и расчетов представлены в табл. 1 .

График зависимости внутреннего квантового выхода от тока, рассчитанной путем деления внешнего квантового выхода на коэффициент вывода излучения, приведен на рис. 2.

Достоверность представленного способа измерения подтверждена путем сравнения полученных результатов измерений с результатами измерений внутреннего квантового выхода способом, приведенным в работе [2], состоящем в измерении ватт-амперной характеристики светодиода, определении токов излучательной и безызлучательной рекомбинаций путем преобразования ватт-амперной характеристики и расчете зависимо- 
Таблица 2. Сравнение результатов измерений внутреннего квантового выхода разными способами

\begin{tabular}{c|c|c|c}
\hline$I, \mathrm{~A}$ & $\begin{array}{c}\text { Результат измерения } \\
\eta(I) \text { предложенным } \\
\text { способом }\end{array}$ & $\begin{array}{c}\text { Результат измерения } \\
\eta(I) \text { способом, } \\
\text { представленным в }[2]\end{array}$ & $\begin{array}{c}\text { Относительная } \\
\text { разница результатов } \\
\text { измерений, \% }\end{array}$ \\
\hline $10 \cdot 10^{-6}$ & 0.312 & 0.322 & 3.1 \\
$50 \cdot 10^{-6}$ & 0.573 & 0.590 & 2.9 \\
$0.7 \cdot 10^{-3}$ & 0.709 & 0.731 & 3.0 \\
$20 \cdot 10^{-3}$ & 0.460 & 0.475 & 3.2
\end{tabular}

сти $\eta(I)$. В табл. 2 представлены результаты измерения внутреннего квантового выхода при токах $I_{1}$ и $I_{2}$ представленным способом и способом, приведенным в работе [2], а также относительная разница результатов измерений.

Полученные разными способами результаты измерений согласуются, при этом относительная разница результатов измерений находится на уровне $3 \%$ во всем диапазоне рабочих токов светодиода.

\section{Заключение}

Представлен способ измерения внутреннего квантового выхода излучения InGaN светодиода. В основе способа измерения лежит АВС-модель рекомбинации носителей заряда в гетероструктуре светодиода. Способ состоит в измерении мощности оптического излучения светодиода и граничных частот электролюминесценции при двух значениях тока, соответствующих диапазону роста токовой зависимости внутренней квантовой эффективности светодиода, при которых влиянием оже-рекомбинации можно пренебречь. Предложенный способ апробирован на примере измерения внутреннего квантового выхода зеленого коммерческого InGaN светодиода. Достоверность подтверждена путем сопоставления результатов измерений с результатами измерений, полученными известным способом. Относительная разница результатов измерений находится на уровне $3 \%$ во всем диапазоне токов.

\section{Финансирование работы}

Работа выполнена в рамках государственного задания при частичной финансовой поддержке РФФИ в рамках научного проекта № 19-07-00562 А.

\section{Конфликт интересов}

Авторы заявляют, что у них нет конфликта интересов.

\section{Список литературы}

[1] J.-I. Shim, D.-S. Shin. Nanophotonics, 7 (10), 1601 (2018). DOI: 10.1515/nanoph-2018-0094

[2] D.-P. Han, K. Yamamoto, S. Ishimoto, M. Iwaya, T. Takeuchi, S. Kamiyama, I. Akasaki. Appl. Phys. Express, 12, 032006 (2019). DOI: 10.7567/1882-0786/aafca2

[3] В.А. Сергеев, А.М. Ходаков, И.В. Фролов. РЭНСИТ, 12 (3), 329 (2020). DOI: 10.17725/rensit.2020.12.301

[4] О.А. Радаев, В.А. Сергеев, И.В. Фролов. Измерительная техника, 8, 42 (2019). DOI: 10.32446/0368-1025it.2019-8-42-46

[5] S. Watanabe, N. Yamada, M. Nagashima, Y. Ueki, C. Sasaki, Y. Yamada, T. Taguchi, K. Tadatomo, H. Okagawa, H. Kudo. Appl. Phys. Lett. 83, 4906 (2003). DOI: 10.1063/1.1633672

[6] G. Chen, M. Craven, A. Kim, A. Munkholm, S. Watanabe, M. Camras, W. Götz, F. Steranka. Phys. Stat. Sol. A, 205 (5), 1086 (2008). DOI: 10.1002/pssa.200778747

[7] I.E. Titkov, D.A. Sannikov, Y.-M. Park, J.-K. Son. AIP Adv., 2, 032117 (2012). DOI: 10.1063/1.4739409

[8] Y. Wang, M. Pan, T. Li. Proc SPIE, 9003, 90030D (2014). DOI: $10.1117 / 12.2040710$

[9] Способ контроля внутреннего квантового выхода полупроводниковых светодиодных гетероструктур на основе GaN. Прудаев И.А., Олешко В.И., Корепанов В.И., Лисицын В.М., Толбанов О.П., Ивонин И.В. Патент 2503024 РФ, МПК G01R 31/26. („Национальный исследовательский Томский гос. ун-т“" (ТГУ). № 2012112682/28; заявл. 03.04.2012; опубл. 7.12.2013. Бюл. № 36)

[10] Способ измерения внутреннего квантового выхода светодиода. Сергеев В.А., Фролов И.В. Патент 2740433 РФ, МПК G01R 31/26. (ИРЭ им. В.А. Котельникова РАН. № 2020116923/28(028230); заявл. 12.05.2020; опубл. 14.01.21. Бюл. № 2)

[11] M. Meneghini, N. Trivellin, G. Meneghesso, E. Zanoni, U. Zehnder, B. Hahn. J. Appl. Phys., 106, 114508 (2009). DOI: $10.1063 / 1.3266014$

[12] P. Tian, P.R. Edwards, M.J. Wallace, R.W. Martin, J.J.D. Mc Kendry, E. Gu, M.D. Dawson, Z.-J. Qiu, C. Jia, Z. Chen, G. Zhang, L. Zheng, R. Liu. J. Phys. D: Appl. Phys., 50, 075101 (2017). DOI: 10.1088/1361-6463/50/7/075101

[13] Ф. Шуберт. Светодиоды (Физматлит, М., 2008) 